\title{
P04-2-10 Poster session
}

\section{Carbonylation of protein phosphatases in hydrogen peroxide or PDGF-BB- treated mouse artery smooth muscle cells}

\author{
Cheng-Ying Hsieh ${ }^{1}$, Chi-Li Chung ${ }^{2}$, Chieh-Min Chen ${ }^{1}$ \\ ${ }^{1}$ Department of Phamacology, Taipei Medical University, Taiwan, ${ }^{2}$ School of Respiratory Therapy, Taipei Medical \\ University, Taiwan
}

Reactive oxygen species (ROS) had been known to influence many signaling events through oxidative modification of proteins. The oxidation of protein phosphatases including protein tyrosine phosphatases (PTPs) and Ser/Thr protein phosphatases (PPases) is particularly important in redox signaling, because the inactivation of protein phosphatases can induce a broad range of signaling pathways. The reversible oxidation and inactivation of PTPs to the sulfenic acid (SOH) state is a well-known redox regulation of signaling transduction. Recently, protein carbonylation also attracted people's attention. This metal-catalyzed protein oxidation was considered to be an irreversible and non-enzymatic modification of proteins. However, a pioneer study has indicated that protein carbonylation/de-carbonylation may be another important mechanism to be involved in redox signaling. Upon stimulation, carbonylated proteins can be decreased subsequent to the peak of carbonylation within short term, and the de-carbonylation process is a proteasome-independent reaction. In this study, we found that the protein phosphatase activities of Ser/Thr PPases and PTPs were both down-regulated in mouse artery smooth muscle cells (ASMCs)-treated by hydrogen peroxide. Moreover, the carbonylation of PP2A and SHP-1 were both determined in hydrogen peroxide-treated VSMCs by using immunoprecipitation assay. On the other hand, the carbonylation/de-carbonylation process was observed in hydrogen peroxide or PDGF-BB-stimulated mouse ASMCs. Nevertheless, the phenomenon of protein de-carbonylation was not observed in mouse ASMCs-stimulated by 4hydroxynonenal. Furthermore, the treatment of MG132, a proteasome inhibitor, could not abolish the carbonylation/decarbonylation process in hydrogen peroxide or PDGF-BB-stimulated mouse ASMCs. The results reveal that carbonylation/de-carbonylation process only occurred in primary protein carbonylation but not in secondary protein carbonylation. This carbonylation/de-carbonylation process is a proteasome-independent signaling pathway. In conclusion, we indicate that the carbonylation/de-carbonylation of protein phosphatases may be a novel redox regulatory pathway in activated ASMCs and ASMC associated diseases. 